\title{
Two Points Taylor's Type Representations for Analytic Complex Functions with Integral Remainders
}

\author{
Silvestru Sever Dragomir
}

\begin{abstract}
In this paper we establish some two point weighted Taylor's expansions for analytic functions $f: D \subseteq \mathbb{C} \rightarrow \mathbb{C}$ defined on a convex domain $D$. Some error bounds for these expansions are also provided. Examples for the complex logarithm and the complex exponential are also given.
\end{abstract}

\section{Introduction}

Let $f: D \subseteq \mathbb{C} \rightarrow \mathbb{C}$ be an analytic function on the convex domain $D$ and $z$, $v \in D$, then we have the following Taylor expansion with integral remainder

$$
\begin{aligned}
f(z)=\sum_{k=0}^{n} \frac{1}{k !} f^{(k)} & (v)(z-v)^{k} \\
& +\frac{1}{n !}(z-v)^{n+1} \int_{0}^{1} f^{(n+1)}[(1-s) v+s z](1-s)^{n} d s
\end{aligned}
$$

for $n \geq 0$, see for instance [13].

In this paper we establish some two point weighted Taylor expansions for analytic functions $f: D \subseteq \mathbb{C} \rightarrow \mathbb{C}$ defined on a convex domain $D$. Some error bounds for these expansions are also provided. Examples for the complex logarithm and the complex exponential are given as well.

Key Words: Taylor's formula, Power series, Logarithmic and exponential functions.

2010 Mathematics Subject Classification: Primary 30B10, 26D15; Secondary 26D10.

Received: 05.09.2020

Accepted: 15.10 .2020 
Consider the function $f(z)=\log (z)$ where $\log (z)=\ln |z|+i \operatorname{Arg}(z)$ and $\operatorname{Arg}(z)$ is such that $-\pi<\operatorname{Arg}(z) \leq \pi$. Log is called the "principal branch" of the complex logarithmic function. The function $f$ is analytic on all of $\mathbb{C}_{\ell}:=\mathbb{C} \backslash\{x+i y: x \leq 0, y=0\}$ and

$$
f^{(k)}(z)=\frac{(-1)^{k-1}(k-1) !}{z^{k}}, k \geq 1, z \in \mathbb{C}_{\ell} .
$$

Using the representation (1) we then have

$$
\begin{aligned}
\log (z)=\log (v)+\sum_{k=1}^{n} \frac{(-1)^{k-1}}{k} & \left(\frac{z-v}{v}\right)^{k} \\
& +(-1)^{n}(z-v)^{n+1} \int_{0}^{1} \frac{(1-s)^{n} d s}{[(1-s) v+s z]^{n+1}}
\end{aligned}
$$

for all $z, v \in \mathbb{C}_{\ell}$ with $(1-s) v+s z \in \mathbb{C}_{\ell}$ for $s \in[0,1]$.

Consider the complex exponential function $f(z)=\exp (z)$, then by (1) we get

$$
\begin{aligned}
\exp (z)=\sum_{k=0}^{n} \frac{1}{k !}(z-v)^{k} \exp (v) & \\
& +\frac{1}{n !}(z-v)^{n+1} \int_{0}^{1}(1-s)^{n} \exp [(1-s) v+s z] d s
\end{aligned}
$$

for all $z, v \in \mathbb{C}$.

For various inequalities related to Taylor expansions for real functions see [1]-[12].

\section{Two Points Taylor Expansions}

We have the following two points Taylor expansion with integral remainder:

Theorem 1. Let $f: D \subseteq \mathbb{C} \rightarrow \mathbb{C}$ be an analytic function on the convex domain $D$ and $z, v, w \in D$, then for all $\lambda \in \mathbb{C}$ we have

$$
\begin{aligned}
f(z) & =(1-\lambda) f(v)+\lambda f(w) \\
& +\sum_{k=1}^{n} \frac{1}{k !}\left[(1-\lambda) f^{(k)}(v)(z-v)^{k}+(-1)^{k} \lambda f^{(k)}(w)(w-z)^{k}\right] \\
& +S_{n, \lambda}(z, v, w),
\end{aligned}
$$


where the remainder $S_{n, \lambda}(z, v, w)$ is given by

$$
\begin{aligned}
& S_{n, \lambda}(z, v, w) \\
& :=\frac{1}{n !}\left[(1-\lambda)(z-v)^{n+1} \int_{0}^{1} f^{(n+1)}[(1-s) v+s z](1-s)^{n} d s\right. \\
& \left.+(-1)^{n+1} \lambda(w-z)^{n+1} \int_{0}^{1} f^{(n+1)}[(1-s) z+s w] s^{n} d s\right] .
\end{aligned}
$$

Proof. If we replace in (1) $v$ by $w$, then we get

$$
\begin{aligned}
& f(z)=\sum_{k=0}^{n} \frac{1}{k !} f^{(k)}(w)(z-w)^{k} \\
& \quad+\frac{1}{n !}(z-w)^{n+1} \int_{0}^{1} f^{(n+1)}[(1-s) w+s z](1-s)^{n} d s \\
& =\sum_{k=0}^{n} \frac{(-1)^{k}}{k !} f^{(k)}(w)(w-z)^{k} \\
& +\frac{(-1)^{n+1}}{n !}(w-z)^{n+1} \int_{0}^{1} f^{(n+1)}[(1-s) w+s z](1-s)^{n} d s \\
& \quad=\sum_{k=0}^{n} \frac{(-1)^{k}}{k !} f^{(k)}(w)(w-z)^{k} \\
& \quad+\frac{(-1)^{n+1}}{n !}(w-z)^{n+1} \int_{0}^{1} f^{(n+1)}[(1-s) z+s w] s^{n} d s .
\end{aligned}
$$

Assume that $\lambda \neq 1,0$. If we multiply (1) by $1-\lambda$ and (6) by $\lambda$ we get the desired representation (4) with the remainder $S_{n, \lambda}(z, v, w)$ given by $(5)$.

If either $\lambda=1$ or $\lambda=0$, then the theorem also holds by the use of Taylor usual expansion.

Remark 1. We observe that for $n=0$ the representation from Theorem 1 becomes

$$
f(z)=(1-\lambda) f(v)+\lambda f(w)+S_{\lambda}(z, v, w),
$$

where the remainder $S_{\lambda}(z, v, w)$ is given by

$$
\begin{aligned}
S_{\lambda}(z, v, w):=(1-\lambda)(z-v) \int_{0}^{1} & f^{\prime}((1-s) v+s z) d s \\
& -\lambda(w-z) \int_{0}^{1} f^{\prime}((1-s) z+s w) d s .
\end{aligned}
$$


Remark 2. If we take in (6) $z=\frac{v+w}{2}$, with $v, w \in D$, then we have for any $\lambda \in \mathbb{C}$ that

$$
\begin{aligned}
f\left(\frac{v+w}{2}\right) & =(1-\lambda) f(v)+\lambda f(w) \\
& +\sum_{k=1}^{n} \frac{1}{2^{k} k !}\left[(1-\lambda) f^{(k)}(v)+(-1)^{k} \lambda f^{(k)}(w)\right](w-v)^{k} \\
& +\tilde{S}_{n, \lambda}(v, w),
\end{aligned}
$$

where the remainder $\tilde{S}_{n, \lambda}(v, w)$ is given by

$$
\begin{aligned}
& \tilde{S}_{n, \lambda}(v, w) \\
& :=\frac{1}{2^{n+1} n !}(w-v)^{n+1}\left[(1-\lambda) \int_{0}^{1} f^{(n+1)}\left((1-s) v+s \frac{v+w}{2}\right)(1-s)^{n} d s\right. \\
& \left.+(-1)^{n+1} \lambda \int_{0}^{1} f^{(n+1)}\left((1-s) \frac{v+w}{2}+s w\right) s^{n} d s\right] .
\end{aligned}
$$

In particular, for $\lambda=\frac{1}{2}$ in (9) we have

$$
\begin{aligned}
f\left(\frac{v+w}{2}\right) & =\frac{f(v)+f(w)}{2} \\
& +\sum_{k=1}^{n} \frac{1}{2^{k+1} k !}\left[f^{(k)}(v)+(-1)^{k} f^{(k)}(w)\right](w-v)^{k} \\
& +\tilde{S}_{n}(v, w),
\end{aligned}
$$

where the remainder $\tilde{S}_{n}(v, w)$ is given by

$$
\begin{aligned}
& \tilde{S}_{n}(v, w) \\
& :=\frac{1}{2^{n+2} n !}(w-v)^{n+1}\left[\int_{0}^{1} f^{(n+1)}\left((1-s) v+s \frac{v+w}{2}\right)(1-s)^{n} d s\right. \\
& \left.+(-1)^{n+1} \int_{0}^{1} f^{(n+1)}\left((1-s) \frac{v+w}{2}+s w\right) s^{n} d s\right] .
\end{aligned}
$$

Now, by the change of variable in (12) we also get the following representation for the remainder $\tilde{S}_{n}(v, w)$ as a single integral

$$
\begin{aligned}
& \tilde{S}_{n}(v, w):=\frac{1}{2^{n+2} n !}(w-v)^{n+1} \\
\times & \int_{0}^{1}\left[f^{(n+1)}\left(s v+(1-s) \frac{v+w}{2}\right)+(-1)^{n+1} f^{(n+1)}\left((1-s) \frac{v+w}{2}+s w\right)\right] s^{n} d s,
\end{aligned}
$$


for $n \geq 0$.

Corollary 2. With the assumptions in Theorem 1 we have for each distinct $z, v, w \in D$ with $w \neq v$

$$
\begin{aligned}
f(z) & =\frac{1}{w-v}[(w-z) f(v)+(z-v) f(w)]+\frac{(w-z)(z-v)}{w-v} \\
& \times \sum_{k=1}^{n} \frac{1}{k !}\left\{(z-v)^{k-1} f^{(k)}(v)+(-1)^{k}(w-z)^{k-1} f^{(k)}(w)\right\} \\
& +L_{n}(z, v, w),
\end{aligned}
$$

where

$$
\begin{aligned}
L_{n}(z, v, w) & :=\frac{(w-z)(z-v)}{n !(w-v)}\left[(z-v)^{n} \int_{0}^{1} f^{(n+1)}((1-s) v+s z)(1-s)^{n} d s\right. \\
& \left.+(-1)^{n+1}(w-z)^{n} \int_{0}^{1} f^{(n+1)}((1-s) z+s w) s^{n} d s\right]
\end{aligned}
$$

and

$$
\begin{aligned}
f(z) & =\frac{1}{w-v}[(z-v) f(v)+(w-z) f(w)] \\
& +\frac{1}{w-v} \sum_{k=1}^{n} \frac{1}{k !}\left\{(z-v)^{k+1} f^{(k)}(v)+(-1)^{k}(w-z)^{k+1} f^{(k)}(w)\right\} \\
& +P_{n}(z, v, w),
\end{aligned}
$$

where

$$
\begin{aligned}
P_{n}(z, v, w) & :=\frac{1}{n !(w-v)}\left[(z-v)^{n+2} \int_{0}^{1} f^{(n+1)}((1-s) v+s z)(1-s)^{n} d s\right. \\
& \left.+(-1)^{n+1}(w-z)^{n+2} \int_{0}^{1} f^{(n+1)}((1-s) z+s w) s^{n} d s\right],
\end{aligned}
$$

respectively.

The proof is obvious, by choosing $\lambda=(z-v) /(w-v)$ and $\lambda=(w-z) /(w-v)$, respectively, in Theorem 1. The details are omitted.

Corollary 3. With the assumption in Theorem 1 we have for each $\lambda \in[0,1]$ and any distinct $v, w \in D$ that

$$
\begin{aligned}
& \quad f((1-\lambda) v+\lambda w)=(1-\lambda) f(v)+\lambda f(w)+\lambda(1-\lambda) \\
& \times \sum_{k=1}^{n} \frac{1}{k !}\left[\lambda^{k-1} f^{(k)}(v)+(-1)^{k}(1-\lambda)^{k-1} f^{(k)}(w)\right](w-v)^{k}+S_{n, \lambda}(v, w),
\end{aligned}
$$


where the remainder $S_{n, \lambda}(v, w)$ is given by

$$
\begin{aligned}
& S_{n, \lambda}(v, w) \\
& \quad:=\frac{1}{n !}(1-\lambda) \lambda(w-v)^{n+1}\left[\lambda^{n} \int_{0}^{1} f^{(n+1)}((1-s \lambda) v+s \lambda w)(1-s)^{n} d s\right. \\
& \left.+(-1)^{n+1}(1-\lambda)^{n} \int_{0}^{1} f^{(n+1)}((1-s-\lambda+s \lambda) v+(\lambda+s-s \lambda) w) s^{n} d s\right] .
\end{aligned}
$$

We also have

$$
\begin{aligned}
& f((1-\lambda) w+\lambda v)=(1-\lambda) f(v)+\lambda f(w) \\
& +\sum_{k=1}^{n} \frac{1}{k !}\left[(1-\lambda)^{k+1} f^{(k)}(v)+(-1)^{k} \lambda^{k+1} f^{(k)}(w)\right](w-v)^{k}+P_{n, \lambda}(v, w)
\end{aligned}
$$

where the remainder $P_{n, \lambda}(v, w)$ is given by

$$
\begin{aligned}
& P_{n, \lambda}(v, w) \\
& \quad:=\frac{1}{n !}(w-v)^{n+1}\left[(1-\lambda)^{n+2} \int_{0}^{1} f^{(n+1)}((1-s+\lambda s) v+(1-\lambda) s w)(1-s)^{n} d s\right. \\
& \left.\quad+(-1)^{n+1} \lambda^{n+2} \int_{0}^{1} f^{(n+1)}((1-s) \lambda v+(1-\lambda+\lambda s) w) s^{n} d s\right] . \quad
\end{aligned}
$$

The case $n=0$ produces the following simple identities for each distinct $z, v, w \in D$ and $\lambda \in \mathbb{C}$

$$
f(z)=(1-\lambda) f(v)+\lambda f(w)+S_{\lambda}(z, v, w),
$$

where the remainder $S_{\lambda}(z, v, w)$ is given by (8).

We then have for each distinct $z, v, w \in D$

$$
f(z)=\frac{1}{w-v}[(w-z) f(v)+(z-v) f(w)]+L(z, v, w)
$$

where

$$
\begin{aligned}
& L(z, v, w) \\
& :=\frac{(w-z)(z-v)}{w-v}\left[\int_{0}^{1} f^{\prime}((1-s) v+s z) d s-\int_{0}^{1} f^{\prime}((1-s) z+s w) d s\right]
\end{aligned}
$$


and

$$
f(z)=\frac{1}{w-v}[(z-v) f(v)+(w-z) f(w)]+P(z, v, w)
$$

where

$$
\begin{aligned}
& P(z, v, w) \\
:= & \frac{1}{w-v}\left[(z-v)^{2} \int_{0}^{1} f^{\prime}((1-s) v+s z) d s-(w-z)^{2} \int_{0}^{1} f^{\prime}((1-s) z+s w) d s\right] .
\end{aligned}
$$

We also have for $\lambda \in[0,1]$

$$
f((1-\lambda) v+\lambda w)=(1-\lambda) f(v)+\lambda f(w)+S_{\lambda}(v, w),
$$

where the remainder $S_{\lambda}(v, w)$ is given by

$$
\begin{aligned}
S_{\lambda}(v, w) & :=(1-\lambda) \lambda(w-v)\left[\int_{0}^{1} f^{\prime}((1-s \lambda) v+s \lambda w) d s\right. \\
& \left.-\int_{0}^{1} f^{\prime}((1-s-\lambda+s \lambda) v+(\lambda+s-s \lambda) w) d s\right]
\end{aligned}
$$

and

$$
f((1-\lambda) w+\lambda v)=(1-\lambda) f(v)+\lambda f(w)+P_{\lambda}(v, w),
$$

where the remainder $P_{\lambda}(v, w)$ is given by

$$
\begin{array}{r}
P_{\lambda}(v, w):=(w-v)\left[(1-\lambda)^{2} \int_{0}^{1} f^{\prime}((1-s+\lambda s) v+(1-\lambda) s w) d s\right. \\
\left.-\lambda^{2} \int_{0}^{1} f^{\prime}((1-s) \lambda v+(1-\lambda+\lambda s) w) d s\right] .
\end{array}
$$

Moreover, if we take in (20) $z=\frac{v+w}{2}$ for each distinct $v, w \in D$ and $\lambda \in \mathbb{C}$, then we have

$$
f\left(\frac{v+w}{2}\right)=(1-\lambda) f(v)+\lambda f(w)+S_{\lambda}(v, w),
$$

where the remainder $S_{\lambda}(v, w)$ is given by

$$
\begin{aligned}
& S_{\lambda}(v, w):=\frac{1}{2}(w-v) \\
& \times\left[(1-\lambda) \int_{0}^{1} f^{\prime}\left((1-s) v+s \frac{v+w}{2}\right) d s-\lambda \int_{0}^{1} f^{\prime}\left((1-s) \frac{v+w}{2}+s w\right) d s\right] .
\end{aligned}
$$


In particular, for $\lambda=\frac{1}{2}$ we have

$$
f\left(\frac{v+w}{2}\right)=\frac{f(v)+f(w)}{2}+S(v, w),
$$

where

$$
\begin{aligned}
& S(v, w):=\frac{1}{4}(w-v) \\
\times & {\left[\int_{0}^{1} f^{\prime}\left((1-s) v+s \frac{v+w}{2}\right) d s-\int_{0}^{1} f^{\prime}\left((1-s) \frac{v+w}{2}+s w\right) d s\right] . }
\end{aligned}
$$

Now, assume that $z, v, w \in D \subset \mathbb{C}_{\ell}$, with $D$ a convex set, then for all $\lambda \in \mathbb{C}$ we have by Theorem 1 for the function $f(z)=\log (z)$ that

$$
\begin{aligned}
\log (z) & =(1-\lambda) \log (v)+\lambda \log (w) \\
& +\sum_{k=1}^{n} \frac{1}{k}\left[(1-\lambda)(-1)^{k-1} \frac{(z-v)^{k}}{v^{k}}-\lambda \frac{(w-z)^{k}}{w^{k}}\right] \\
& +S_{n, \lambda}(z, v, w),
\end{aligned}
$$

where the remainder $\Lambda_{n, \lambda}(z, v, w)$ is given by

$$
\begin{aligned}
\Lambda_{n, \lambda}(z, v, w) & :=(1-\lambda)(z-v)^{n+1}(-1)^{n} \int_{0}^{1} \frac{(1-s)^{n}}{((1-s) v+s z)^{n+1}} d s \\
& -\lambda(w-z)^{n+1} \int_{0}^{1} \frac{s^{n}}{((1-s) z+s w)^{n+1}} d s
\end{aligned}
$$

for $n \geq 0$.

Consider the function $f: \mathbb{C} \longrightarrow \mathbb{C}, f(z)=\exp z$, then for $z, v, w \in \mathbb{C}$ we have by Theorem 1 that

$$
\begin{aligned}
\exp z & =(1-\lambda) \exp v+\lambda \exp w \\
& +\sum_{k=1}^{n} \frac{1}{k !}\left[(1-\lambda)(z-v)^{k} \exp v+(-1)^{k} \lambda(w-z)^{k} \exp w\right] \\
& +\Theta_{n, \lambda}(z, v, w),
\end{aligned}
$$

where the remainder $\Theta_{n, \lambda}(z, v, w)$ is given by

$$
\begin{aligned}
& \Theta_{n, \lambda}(z, v, w) \\
& :=\frac{1}{n !}\left[(1-\lambda)(z-v)^{n+1} \int_{0}^{1} \exp ((1-s) v+s z)(1-s)^{n} d s\right. \\
& \left.+(-1)^{n+1} \lambda(w-z)^{n+1} \int_{0}^{1} \exp ((1-s) z+s w) s^{n} d s\right] .
\end{aligned}
$$


for $n \geq 0$ and for all $\lambda \in \mathbb{C}$.

\section{Some Inequalities}

We can state now some results concerning error bounds in approximating an analytic function by two points Taylor expansion:

Theorem 4. Let $f: D \subseteq \mathbb{C} \rightarrow \mathbb{C}$ be an analytic function on the convex domain $D$ and $z, v, w \in D$, then for all $\lambda \in \mathbb{C}$ we have

$$
\begin{aligned}
f(z) & =(1-\lambda) f(v)+\lambda f(w) \\
& +\sum_{k=1}^{n} \frac{1}{k !}\left[(1-\lambda) f^{(k)}(v)(z-v)^{k}+(-1)^{k} \lambda f^{(k)}(w)(w-z)^{k}\right] \\
& +S_{n, \lambda}(z, v, w),
\end{aligned}
$$

and the remainder $S_{n, \lambda}(z, v, w)$ satisfies the inequalities

$$
\begin{aligned}
& \left|S_{n, \lambda}(z, v, w)\right| \\
& \leq \frac{1}{n !}|1-\lambda||z-v|^{n+1} \int_{0}^{1}\left|f^{(n+1)}((1-s) v+s z)\right|(1-s)^{n} d s \\
& +\frac{1}{n !}|\lambda||w-z|^{n+1} \int_{0}^{1}\left|f^{(n+1)}((1-s) z+s w)\right| s^{n} d s \\
& \leq \frac{1}{n !}|1-\lambda||z-v|^{n+1}\left\{\begin{array}{l}
\frac{1}{n+1} \sup _{s \in[0,1]}\left|f^{(n+1)}((1-s) v+s z)\right| \\
\frac{1}{(q n+1)^{1 / q}}\left(\int_{0}^{1}\left|f^{(n+1)}((1-s) v+s z)\right|^{p} d s\right)^{1 / p} \\
\text { where } p, q>1 \text { with } \frac{1}{p}+\frac{1}{q}=1 \\
\int_{0}^{1}\left|f^{(n+1)}((1-s) v+s z)\right| d s
\end{array}\right. \\
& +\frac{1}{n !}|\lambda||w-z|^{n+1}\left\{\begin{array}{l}
\frac{1}{n+1} \sup _{s \in[0,1]}\left|f^{(n+1)}((1-s) z+s w)\right| \\
\frac{1}{(q n+1)^{1 / q}}\left(\int_{0}^{1}\left|f^{(n+1)}((1-s) z+s w)\right|^{p} d s\right)^{1 / p} \\
\text { where } p, q>1 \text { with } \frac{1}{p}+\frac{1}{q}=1 \\
\int_{0}^{1}\left|f^{(n+1)}((1-s) z+s w)\right| d s
\end{array}\right.
\end{aligned}
$$

for $n \geq 0$. 
Proof. Taking the modulus in the representation (5), we get

$$
\begin{aligned}
& \left|S_{n, \lambda}(z, v, w)\right| \\
& \leq \frac{1}{n !}\left[\left|(1-\lambda)(z-v)^{n+1} \int_{0}^{1} f^{(n+1)}((1-s) v+s z)(1-s)^{n} d s\right|\right. \\
& \left.+\left|\lambda(w-z)^{n+1} \int_{0}^{1} f^{(n+1)}((1-s) z+s w) s^{n} d s\right|\right] \\
& \leq|1-\lambda||z-v|^{n+1} \int_{0}^{1}\left|f^{(n+1)}((1-s) v+s z)\right|(1-s)^{n} d s \\
& +|\lambda||w-z|^{n+1} \int_{0}^{1}\left|f^{(n+1)}((1-s) z+s w)\right| s^{n} d s .
\end{aligned}
$$

By Hölder's integral inequality we have

$$
\begin{aligned}
& \int_{0}^{1}\left|f^{(n+1)}((1-s) v+s z)\right|(1-s)^{n} d s \\
& \leq\left\{\begin{array}{l}
\sup _{s \in[0,1]}\left|f^{(n+1)}((1-s) v+s z)\right| \int_{0}^{1}(1-s)^{n} d s \\
\left(\int_{0}^{1}\left|f^{(n+1)}((1-s) v+s z)\right|^{p} d s\right)^{1 / p}\left(\int_{0}^{1}(1-s)^{q n} d s\right)^{1 / q} \\
\text { where } p, q>1 \text { with } \frac{1}{p}+\frac{1}{q}=1 \\
\sup _{s \in[0,1]}\left\{(1-s)^{n}\right\} \int_{0}^{1}\left|f^{(n+1)}((1-s) v+s z)\right| d s
\end{array}\right. \\
& =\left\{\begin{array}{l}
\frac{1}{n+1} \sup _{s \in[0,1]}\left|f^{(n+1)}((1-s) v+s z)\right| \\
\frac{1}{(q n+1)^{1 / q}}\left(\int_{0}^{1}\left|f^{(n+1)}((1-s) v+s z)\right|^{p} d s\right)^{1 / p} \\
\text { where } p, q>1 \text { with } \frac{1}{p}+\frac{1}{q}=1
\end{array}\right. \\
& \int_{0}^{1}\left|f^{(n+1)}((1-s) v+s z)\right| d s
\end{aligned}
$$


and

$$
\begin{aligned}
& \int_{0}^{1}\left|f^{(n+1)}((1-s) z+s w)\right| s^{n} d s \\
& \leq\left\{\begin{array}{l}
\sup _{s \in[0,1]}\left|f^{(n+1)}((1-s) z+s w)\right| \int_{0}^{1} s^{n} d s \\
\left(\int_{0}^{1}\left|f^{(n+1)}((1-s) z+s w)\right|^{p} d s\right)^{1 / p}\left(\int_{0}^{1} s^{q n} d s\right)^{1 / q}
\end{array}\right. \\
& \text { where } p, q>1 \text { with } \frac{1}{p}+\frac{1}{q}=1 \\
& \sup _{s \in[0,1]}\left\{s^{n}\right\} \int_{0}^{1}\left|f^{(n+1)}((1-s) z+s w)\right| d s \\
& =\left\{\begin{array}{l}
\frac{1}{n+1} \sup _{s \in[0,1]}\left|f^{(n+1)}((1-s) z+s w)\right| \\
\frac{1}{(q n+1)^{1 / q}}\left(\int_{0}^{1}\left|f^{(n+1)}((1-s) z+s w)\right|^{p} d s\right)^{1 / p} \\
\text { where } p, q>1 \text { with } \frac{1}{p}+\frac{1}{q}=1
\end{array}\right. \\
& \left(\int_{0}^{1}\left|f^{(n+1)}((1-s) z+s w)\right| d s,\right.
\end{aligned}
$$

which proves the second inequality in (38).

Corollary 5. With the assumptions of Theorem 4 and if

$$
\left\|f^{(n+1)}\right\|_{D, \infty}:=\sup _{y \in D}\left|f^{(n+1)}(y)\right|<\infty
$$

then we have the simple bound

$$
\left|S_{n, \lambda}(z, v, w)\right| \leq \frac{1}{(n+1) !}\left\|f^{(n+1)}\right\|_{D, \infty}\left(|1-\lambda||z-v|^{n+1}+|\lambda||w-z|^{n+1}\right)
$$

for $n \geq 0$.

Remark 3. If we take $z=\frac{v+w}{2}$, with $v, w \in D$, then we have for any $\lambda \in \mathbb{C}$ that

$$
\begin{aligned}
f\left(\frac{v+w}{2}\right) & =(1-\lambda) f(v)+\lambda f(w) \\
& +\sum_{k=1}^{n} \frac{1}{2^{k} k !}\left[(1-\lambda) f^{(k)}(v)+(-1)^{k} \lambda f^{(k)}(w)\right](w-v)^{k} \\
& +\tilde{S}_{n, \lambda}(v, w)
\end{aligned}
$$


and if $\left\|f^{(n+1)}\right\|_{D, \infty}:=\sup _{y \in D}\left|f^{(n+1)}(y)\right|<\infty$, then by (40) we get

$$
\left|\tilde{S}_{n, \lambda}(v, w)\right| \leq \frac{1}{2^{n+1}(n+1) !}\left\|f^{(n+1)}\right\|_{D, \infty}(|1-\lambda|+|\lambda|)|w-v|^{n+1}
$$

or any $\lambda \in \mathbb{C}$.

In particular, if $\lambda=\frac{1}{2}$, then we have

$$
\begin{aligned}
f\left(\frac{v+w}{2}\right) & =\frac{f(v)+f(w)}{2} \\
& +\sum_{k=1}^{n} \frac{1}{2^{k+1} k !}\left[f^{(k)}(v)+(-1)^{k} f^{(k)}(w)\right](w-v)^{k} \\
& +\tilde{S}_{n}(v, w)
\end{aligned}
$$

and the remainder $\tilde{S}_{n}(v, w)$ satisfies the bound

$$
\left|\tilde{S}_{n}(v, w)\right| \leq \frac{1}{2^{n+1}(n+1) !}\left\|f^{(n+1)}\right\|_{D, \infty}|w-v|^{n+1}
$$

for $n \geq 0$.

Remark 4. The case $n=0$ provides some simple inequalities as follows

$$
\begin{aligned}
& \quad|f(z)-(1-\lambda) f(v)-\lambda f(w)| \\
& \leq|1-\lambda||z-v| \int_{0}^{1}\left|f^{\prime}((1-s) v+s z)\right| d s+|\lambda||w-z| \int_{0}^{1}\left|f^{\prime}((1-s) z+s w)\right| d s \\
& \leq|1-\lambda||z-v|\left\{\begin{array}{l}
\sup _{s \in[0,1]}\left|f^{\prime}((1-s) v+s z)\right| \\
\left(\int_{0}^{1}\left|f^{\prime}((1-s) v+s z)\right|^{p} d s\right)^{1 / p} \\
w h e r e p>1 \\
\int_{0}^{1}\left|f^{\prime}((1-s) v+s z)\right| d s
\end{array}\right. \\
& +\quad|\lambda||w-z|\left\{\begin{array}{l}
\sup _{s \in[0,1]}\left|f^{\prime}((1-s) z+s w)\right| \\
\left(\int_{0}^{1}\left|f^{\prime}((1-s) z+s w)\right|^{p} d s\right)^{1 / p} \\
\text { where } p>1 \\
\int_{0}^{1}\left|f^{\prime}((1-s) z+s w)\right| d s
\end{array}\right.
\end{aligned}
$$


where $f: D \subseteq \mathbb{C} \rightarrow \mathbb{C}$ is an analytic function on the convex domain $D, z, v$, $w \in D$ and $\lambda \in \mathbb{C}$.

If

$$
\left\|f^{\prime}\right\|_{D, \infty}:=\sup _{y \in D}\left|f^{\prime}(y)\right|<\infty
$$

then we have the simple bound

$$
|f(z)-(1-\lambda) f(v)-\lambda f(w)| \leq\left\|f^{\prime}\right\|_{D, \infty}(|1-\lambda||z-v|+|\lambda||w-z|)
$$

for $z, v, w \in D$ and $\lambda \in \mathbb{C}$.

If we take $z=\frac{v+w}{2}$, with $v, w \in D$, then we have for any $\lambda \in \mathbb{C}$ that

$$
\left|f\left(\frac{v+w}{2}\right)-(1-\lambda) f(v)-\lambda f(w)\right| \leq \frac{1}{2}\left\|f^{\prime}\right\|_{D, \infty}(|1-\lambda|+|\lambda|)|w-v|,
$$

which for $\lambda=\frac{1}{2}$ gives the simple inequality

$$
\left|f\left(\frac{v+w}{2}\right)-\frac{f(v)+f(w)}{2}\right| \leq \frac{1}{2}\left\|f^{\prime}\right\|_{D, \infty}|w-v| .
$$

If $n$ is even, namely $n=2 m, m \geq 0$, then by (11) we have the representation

$$
\begin{aligned}
f\left(\frac{v+w}{2}\right) & =\frac{f(v)+f(w)}{2} \\
& +\sum_{k=1}^{2 m} \frac{1}{2^{k+1} k !}\left[f^{(k)}(v)+(-1)^{k} f^{(k)}(w)\right](w-v)^{k} \\
& +\tilde{S}_{2 m}(v, w)
\end{aligned}
$$

where the remainder $\tilde{S}_{2 m}(v, w)$ is given by (13) as

$$
\begin{aligned}
\tilde{S}_{2 m}(v, w):= & \frac{1}{2^{2 m+2}(2 m) !}(w-v)^{2 m+1} \\
\times & \int_{0}^{1}\left[f^{(2 m+1)}\left(s v+(1-s) \frac{v+w}{2}\right)-f^{(2 m+1)}\left((1-s) \frac{v+w}{2}+s w\right)\right] s^{2 m} d s,
\end{aligned}
$$

We also have the following result:

Theorem 6. Let $f: D \subseteq \mathbb{C} \rightarrow \mathbb{C}$ be an analytic function on the convex domain $D$ and $v, w \in D$. Let $m \geq 0$ and assume that

$$
\left|f^{(2 m+1)}(z)-f^{(2 m+1)}(y)\right| \leq L_{2 m+1}|z-y| \text { for all } z, y \in D
$$


for some $L_{2 m+1}>0$, namely that $f^{(2 m+1)}$ is Lipschitzian on D. Then we have the representation (47) and the remainder $\tilde{S}_{2 m}(v, w)$ satisfies the bound

$$
\left|\tilde{S}_{2 m}(v, w)\right| \leq \frac{1}{2^{2 m+2}(2 m+2)(2 m) !}|w-v|^{2 m+2} L_{2 m+1}
$$

Proof. By taking the modulus in (48), we have

$$
\begin{gathered}
\left|\tilde{S}_{2 m}(v, w)\right| \leq \frac{1}{2^{2 m+2}(2 m) !}|w-v|^{2 m+1} \\
\times \int_{0}^{1}\left|f^{(2 m+1)}\left(s v+(1-s) \frac{v+w}{2}\right)-f^{(2 m+1)}\left((1-s) \frac{v+w}{2}+s w\right)\right| s^{2 m} d s \\
\quad \leq \frac{1}{2^{2 m+2}(2 m) !}|w-v|^{2 m+1} \\
\times L_{2 m+1} \int_{0}^{1}\left|s v+(1-s) \frac{v+w}{2}-(1-s) \frac{v+w}{2}-s w\right| s^{2 m} d s \\
=\frac{1}{2^{2 m+2}(2 m) !}|w-v|^{2 m+2} L_{2 m+1} \int_{0}^{1} s^{2 m+1} d s \\
=\frac{1}{2^{2 m+2}(2 m+2)(2 m) !}|w-v|^{2 m+2} L_{2 m+1}
\end{gathered}
$$

which proves the desired result (50).

Corollary 7. Let $f: D \subseteq \mathbb{C} \rightarrow \mathbb{C}$ be an analytic function on the convex domain $D$ and $v, w \in D$. Assume that

$$
\left|f^{\prime}(z)-f^{\prime}(y)\right| \leq L|z-y| \text { for all } z, y \in D
$$

for some $L>0$. Then we have the inequality

$$
\left|f\left(\frac{v+w}{2}\right)-\frac{f(v)+f(w)}{2}\right| \leq \frac{1}{8} L|w-v|^{2} .
$$

\section{Inequalities for Convex Derivatives in Absolute Value}

We have:

Theorem 8. Let $f: D \subseteq \mathbb{C} \rightarrow \mathbb{C}$ be an analytic function on the convex domain $D$ and such that for a given $n \geq 0,\left|f^{(n+1)}\right|$ is convex on $D$. If $z, v$, 
$w \in D$, then for all $\lambda \in \mathbb{C}$ we have

$$
\begin{aligned}
f(z) & =(1-\lambda) f(v)+\lambda f(w) \\
& +\sum_{k=1}^{n} \frac{1}{k !}\left[(1-\lambda) f^{(k)}(v)(z-v)^{k}+(-1)^{k} \lambda f^{(k)}(w)(w-z)^{k}\right] \\
& +S_{n, \lambda}(z, v, w),
\end{aligned}
$$

and the remainder $S_{n, \lambda}(z, v, w)$ satisfies the inequality

$$
\begin{aligned}
& \left|S_{n, \lambda}(z, v, w)\right| \leq \frac{1}{n !(n+2)}\left[|1-\lambda||z-v|^{n+1}\left|f^{(n+1)}(v)\right|\right. \\
& +\frac{1}{(n+1)}\left[|1-\lambda||z-v|^{n+1}+|\lambda||w-z|^{n+1}\right]\left|f^{(n+1)}(z)\right| \\
& \left.+|\lambda||w-z|^{n+1}\left|f^{(n+1)}(w)\right|\right] .
\end{aligned}
$$

Proof. Using the representation (5), we get

$$
\begin{aligned}
& \left|S_{n, \lambda}(z, v, w)\right| \\
& \leq \frac{1}{n !}\left[|1-\lambda||z-v|^{n+1}\left|\int_{0}^{1} f^{(n+1)}((1-s) v+s z)(1-s)^{n} d s\right|\right. \\
& \left.+|\lambda||w-z|^{n+1}\left|\int_{0}^{1} f^{(n+1)}((1-s) z+s w) s^{n} d s\right|\right] \\
& \leq \frac{1}{n !}\left[|1-\lambda||z-v|^{n+1} \int_{0}^{1}\left|f^{(n+1)}((1-s) v+s z)\right|(1-s)^{n} d s\right. \\
& \left.+|\lambda||w-z|^{n+1} \int_{0}^{1}\left|f^{(n+1)}((1-s) z+s w)\right| s^{n} d s\right] \\
& =: A_{n}(\lambda, w)
\end{aligned}
$$


By the convexity of $\left|f^{(n+1)}\right|$ we have

$$
\begin{aligned}
& \int_{0}^{1}\left|f^{(n+1)}((1-s) v+s z)\right|(1-s)^{n} d s \\
& \leq \int_{0}^{1}\left[(1-s)\left|f^{(n+1)}(v)\right|+s\left|f^{(n+1)}(z)\right|\right](1-s)^{n} d s \\
& =\left|f^{(n+1)}(v)\right| \int_{0}^{1}(1-s)^{n+1} d s+\left|f^{(n+1)}(z)\right| \int_{0}^{1} s(1-s)^{n} d s \\
& =\frac{1}{n+2}\left|f^{(n+1)}(v)\right|+\left|f^{(n+1)}(z)\right| \int_{0}^{1}(1-s) s^{n} d s \\
& =\frac{1}{n+2}\left|f^{(n+1)}(v)\right|+\left|f^{(n+1)}(z)\right|\left(\frac{1}{n+1}-\frac{1}{n+2}\right) \\
& =\frac{1}{n+2}\left|f^{(n+1)}(v)\right|+\frac{1}{(n+1)(n+2)}\left|f^{(n+1)}(z)\right|
\end{aligned}
$$

and

$$
\begin{aligned}
& \int_{0}^{1}\left|f^{(n+1)}((1-s) z+s w)\right| s^{n} d s \\
& \leq \int_{0}^{1}\left[(1-s)\left|f^{(n+1)}(z)\right|+s\left|f^{(n+1)}(w)\right|\right] s^{n} d s \\
& =\left|f^{(n+1)}(z)\right| \int_{0}^{1}(1-s) s^{n} d s+\left|f^{(n+1)}(w)\right| \int_{0}^{1} s^{n+1} d s \\
& =\frac{1}{(n+1)(n+2)}\left|f^{(n+1)}(z)\right|+\frac{1}{n+2}\left|f^{(n+1)}(w)\right| .
\end{aligned}
$$

Therefore,

$$
\begin{aligned}
& A_{n}(\lambda, w) \\
& \leq \frac{1}{n !}\left[|1-\lambda||z-v|^{n+1}\left[\frac{1}{n+2}\left|f^{(n+1)}(v)\right|+\frac{1}{(n+1)(n+2)}\left|f^{(n+1)}(z)\right|\right]\right. \\
& \left.+|\lambda||w-z|^{n+1}\left[\frac{1}{(n+1)(n+2)}\left|f^{(n+1)}(z)\right|+\frac{1}{n+2}\left|f^{(n+1)}(w)\right|\right]\right]
\end{aligned}
$$




$$
\begin{aligned}
& =\frac{1}{n !(n+2)}\left[|1-\lambda||z-v|^{n+1}\left[\left|f^{(n+1)}(v)\right|+\frac{1}{(n+1)}\left|f^{(n+1)}(z)\right|\right]\right. \\
& \left.+|\lambda||w-z|^{n+1}\left[\frac{1}{(n+1)}\left|f^{(n+1)}(z)\right|+\left|f^{(n+1)}(w)\right|\right]\right] \\
& =\frac{1}{n !(n+2)}\left[|1-\lambda||z-v|^{n+1}\left|f^{(n+1)}(v)\right|\right. \\
& +\frac{1}{(n+1)}\left[|1-\lambda||z-v|^{n+1}+|\lambda||w-z|^{n+1}\right]\left|f^{(n+1)}(z)\right| \\
& \left.+|\lambda||w-z|^{n+1}\left|f^{(n+1)}(w)\right|\right]
\end{aligned}
$$

which together with (55) produce the desired result (55).

Remark 5. Assume that for a given $n \geq 0,\left|f^{(n+1)}\right|$ is convex on D. If we take in (53) $z=\frac{v+w}{2}$, with $v, w \in D$, then we have for any $\lambda \in \mathbb{C}$ that

$$
\begin{aligned}
f\left(\frac{v+w}{2}\right) & =(1-\lambda) f(v)+\lambda f(w) \\
& +\sum_{k=1}^{n} \frac{1}{2^{k} k !}\left[(1-\lambda) f^{(k)}(v)+(-1)^{k} \lambda f^{(k)}(w)\right](w-v)^{k} \\
& +\tilde{S}_{n, \lambda}(v, w),
\end{aligned}
$$

where the remainder $\tilde{S}_{n, \lambda}(v, w)$ satisfies the bound

$$
\begin{aligned}
& \left|\tilde{S}_{n, \lambda}(v, w)\right| \leq \frac{1}{2^{n+1} n !(n+2)}|w-v|^{n+1}\left[|1-\lambda|\left|f^{(n+1)}(v)\right|\right. \\
& \left.\quad+\frac{1}{(n+1)}[|1-\lambda|+|\lambda|]\left|f^{(n+1)}\left(\frac{v+w}{2}\right)\right|+|\lambda|\left|f^{(n+1)}(w)\right|\right] .
\end{aligned}
$$

In particular, for $\lambda=\frac{1}{2}$ in (57) we have

$$
\begin{aligned}
f\left(\frac{v+w}{2}\right) & =\frac{f(v)+f(w)}{2} \\
& +\sum_{k=1}^{n} \frac{1}{2^{k+1} k !}\left[f^{(k)}(v)+(-1)^{k} f^{(k)}(w)\right](w-v)^{k} \\
& +\tilde{S}_{n}(v, w)
\end{aligned}
$$


where the remainder $\tilde{S}_{n}(v, w)$ satisfies the bound

$$
\begin{aligned}
&\left|\tilde{S}_{n}(v, w)\right| \leq \frac{1}{2^{n+1} n !(n+2)}|w-v|^{n+1} {\left[\frac{1}{2}\left|f^{(n+1)}(v)\right|\right.} \\
&\left.+\frac{1}{(n+1)}\left|f^{(n+1)}\left(\frac{v+w}{2}\right)\right|+\frac{1}{2}\left|f^{(n+1)}(w)\right|\right] .
\end{aligned}
$$

Corollary 9. With the assumption in Theorem 8 we have for each $\lambda \in[0,1]$ and any distinct $v, w \in D$ that

$$
\begin{aligned}
& f((1-\lambda) v+\lambda w)=(1-\lambda) f(v)+\lambda f(w)+\lambda(1-\lambda) \\
& \times \sum_{k=1}^{n} \frac{1}{k !}\left[\lambda^{k-1} f^{(k)}(v)+(-1)^{k}(1-\lambda)^{k-1} f^{(k)}(w)\right](w-v)^{k}+S_{n, \lambda}(v, w),
\end{aligned}
$$

where the remainder $S_{n, \lambda}(v, w)$ satisfies the bound

$$
\begin{gathered}
\left|S_{n, \lambda}(v, w)\right| \leq \frac{1}{n !(n+2)}(1-\lambda) \lambda|w-v|^{n+1}\left[\lambda^{n}\left|f^{(n+1)}(v)\right|\right. \\
\left.+\frac{1}{(n+1)}\left[\lambda^{n}+(1-\lambda)^{n}\right]\left|f^{(n+1)}((1-\lambda) v+\lambda w)\right|+(1-\lambda)^{n}\left|f^{(n+1)}(w)\right|\right] .
\end{gathered}
$$

We also have

$$
\begin{aligned}
& f((1-\lambda) w+\lambda v)=(1-\lambda) f(v)+\lambda f(w) \\
+ & \sum_{k=1}^{n} \frac{1}{k !}\left[(1-\lambda)^{k+1} f^{(k)}(v)+(-1)^{k} \lambda^{k+1} f^{(k)}(w)\right](w-v)^{k}+P_{n, \lambda}(v, w),
\end{aligned}
$$

where the remainder $P_{n, \lambda}(v, w)$ satisfies the bound

$$
\begin{aligned}
& \left|P_{n, \lambda}(v, w)\right| \leq \frac{1}{n !(n+2)}|w-v|^{n+1}\left[(1-\lambda)^{n+2}\left|f^{(n+1)}(v)\right|\right. \\
+ & \left.\frac{1}{(n+1)}\left[(1-\lambda)^{n+2}+\lambda^{n+2}\right]\left|f^{(n+1)}((1-\lambda) w+\lambda v)\right|+\lambda^{n+2}\left|f^{(n+1)}(w)\right|\right] .
\end{aligned}
$$

For $n=0$, namely if $\left|f^{\prime}\right|$ is convex on $D$, then by (54) we get

$$
\begin{aligned}
\mid f(z)- & (1-\lambda) f(v)-\lambda f(w) \mid \leq \frac{1}{2}\left[|1-\lambda||z-v|\left|f^{\prime}(v)\right|\right. \\
& \left.+[|1-\lambda||z-v|+|\lambda||w-z|]\left|f^{\prime}(z)\right|+|\lambda||w-z|\left|f^{\prime}(w)\right|\right],
\end{aligned}
$$


for $z, v, w \in D$ and for all $\lambda \in \mathbb{C}$.

From (57) we get

$$
\begin{array}{r}
\left|f\left(\frac{v+w}{2}\right)-(1-\lambda) f(v)-\lambda f(w)\right| \leq \frac{1}{4}|w-v|\left[|1-\lambda|\left|f^{\prime}(v)\right|\right. \\
\left.+[|1-\lambda|+|\lambda|]\left|f^{\prime}\left(\frac{v+w}{2}\right)\right|+|\lambda|\left|f^{\prime}(w)\right|\right]
\end{array}
$$

for $v, w \in D$ and for all $\lambda \in \mathbb{C}$.

In particular, for $\lambda=\frac{1}{2}$ we get

$$
\begin{aligned}
& \left|f\left(\frac{v+w}{2}\right)-\frac{f(v)+f(w)}{2}\right| \\
& \leq \frac{1}{8}|w-v|\left[\left|f^{\prime}(v)\right|+2\left|f^{\prime}\left(\frac{v+w}{2}\right)\right|+\left|f^{\prime}(w)\right|\right]
\end{aligned}
$$

for $v, w \in D$.

\section{Examples for Logarithm and Exponential}

Consider the function $f(z)=\log (z)$ where $\log (z)=\ln |z|+i \operatorname{Arg}(z)$ and $\operatorname{Arg}(z)$ is such that $-\pi<\operatorname{Arg}(z) \leq \pi$. Log is called the "principal branch" of the complex logarithmic function. The function $f$ is analytic on all of $\mathbb{C}_{\ell}:=\mathbb{C} \backslash\{x+i y: x \leq 0, y=0\}$ and

$$
f^{(k)}(z)=\frac{(-1)^{k-1}(k-1) !}{z^{k}}, k \geq 1, z \in \mathbb{C}_{\ell} .
$$

Let $D$ be a convex domain in $\mathbb{C}_{\ell}$ an assume that $d_{D}:=\inf _{z \in D}|z|$ is a positive and finite number. If $z, v, w \in D \subset \mathbb{C}_{\ell}$, then by the representation (17) and the inequality (40)

$$
\begin{aligned}
& \mid \log (z)-(1-\lambda) \log (v)-\lambda \log (w) \\
&-\sum_{k=1}^{n} \frac{1}{k}\left[(1-\lambda)(-1)^{k-1} \frac{(z-v)^{k}}{v^{k}}-\lambda \frac{(w-z)^{k}}{w^{k}}\right] \mid \\
& \leq \frac{1}{(n+1) d_{D}^{n+1}}\left(|1-\lambda||z-v|^{n+1}+|\lambda||w-z|^{n+1}\right)
\end{aligned}
$$

for $n \geq 1$ and for $n=0$ we have

$$
|\log (z)-(1-\lambda) \log (v)-\lambda \log (w)| \leq \frac{1}{d_{D}}(|1-\lambda||z-v|+|\lambda||w-z|),
$$


for all $\lambda \in \mathbb{C}$.

If $\lambda=\tau \in[0,1]$, then by (67) we get

$$
\begin{aligned}
& \mid \log (z)-(1-\tau) \log (v)-\tau \log (w) \\
& \quad-\sum_{k=1}^{n} \frac{1}{k}\left[(1-\tau)(-1)^{k-1} \frac{(z-v)^{k}}{v^{k}}-\tau \frac{(w-z)^{k}}{w^{k}}\right] \mid \\
& \leq \frac{1}{(n+1) d_{D}^{n+1}}\left((1-\tau)|z-v|^{n+1}+\tau|w-z|^{n+1}\right) \\
& \quad \leq \frac{1}{(n+1) d_{D}^{n+1}} \max \left\{|z-v|^{n+1},|w-z|^{n+1}\right\}
\end{aligned}
$$

and for $\tau=\frac{1}{2}$ we get

$$
\begin{aligned}
\mid \log (z)-\frac{\log (v)+\log (w)}{2} & \\
-\frac{1}{2} \sum_{k=1}^{n} \frac{1}{k} & {\left[(-1)^{k-1} \frac{(z-v)^{k}}{v^{k}}-\frac{(w-z)^{k}}{w^{k}}\right] \mid } \\
& \leq \frac{1}{2(n+1) d_{D}^{n+1}}\left(|z-v|^{n+1}+|w-z|^{n+1}\right)
\end{aligned}
$$

for $z, v, w \in D \subset \mathbb{C}_{\ell}$.

Moreover, if we take $z=\frac{v+w}{2}$ in (70), then we get

$$
\begin{aligned}
\mid \log \left(\frac{v+w}{2}\right)- & \frac{\log (v)+\log (w)}{2} \\
& -\frac{1}{2} \sum_{k=1}^{n} \frac{1}{2^{k} k}\left[\frac{(-1)^{k-1}}{v^{k}}-\frac{1}{w^{k}}\right](w-v)^{k} \mid \\
& \leq \frac{1}{2^{n+1}(n+1) d_{D}^{n+1}}|w-v|^{n+1}
\end{aligned}
$$

for $v, w \in D \subset \mathbb{C}_{\ell}$.

The case $n=0$ gives that

$$
\left|\log \left(\frac{v+w}{2}\right)-\frac{\log (v)+\log (w)}{2}\right| \leq \frac{1}{2 d_{D}}|w-v|
$$

for $v, w \in D \subset \mathbb{C}_{\ell}$.

For $f(z)=\log (z), z \in D \subset \mathbb{C}_{\ell}$, we have

$$
\left|f^{\prime}(z)-f^{\prime}(w)\right|=\left|\frac{1}{z}-\frac{1}{w}\right|=\frac{|w-v|}{|z||w|} \leq \frac{1}{d_{D}^{2}}|w-v|
$$


showing that $f^{\prime}$ is Lipschitzian on $D$ with the constant $L=\frac{1}{d_{D}^{2}}$.

By the inequality (52) we then get

$$
\left|\log \left(\frac{v+w}{2}\right)-\frac{\log (v)+\log (w)}{2}\right| \leq \frac{1}{8 d_{D}^{2}}|w-v|^{2},
$$

for $v, w \in D \subset \mathbb{C}_{\ell}$.

Now consider the exponential function $f(z)=\exp z$. Then

$$
|\exp z|=\exp (\operatorname{Re} z)
$$

and

$$
|\exp ((1-t) z+t w)| \leq(1-t)|\exp z|+t|\exp w|
$$

for any $z, w \in \mathbb{C}$ and $t \in[0,1]$, showing that $f$ is convex in absolute value.

Now let $D$ be a convex domain in $\mathbb{C}$ and assume that $E_{D}:=\sup _{z \in D}[\exp (\operatorname{Re} z)]<$ $\infty$. If we use the representation (35) and the inequality (40), we have

$$
\begin{aligned}
\mid \exp z-(1-\lambda) \exp v-\lambda \exp w & \\
-\sum_{k=1}^{n} \frac{1}{k !}[ & \left.(1-\lambda)(z-v)^{k} \exp v+(-1)^{k} \lambda(w-z)^{k} \exp w\right] \mid \\
& \leq \frac{1}{(n+1) !} E_{D}\left(|1-\lambda||z-v|^{n+1}+|\lambda||w-z|^{n+1}\right)
\end{aligned}
$$

for all $z, v, w \in D \subset \mathbb{C}$ and $n \geq 1$.

For $n=0$ we have the simpler inequality

$$
|\exp z-(1-\lambda) \exp v-\lambda \exp w| \leq E_{D}(|1-\lambda||z-v|+|\lambda||w-z|)
$$

for all $z, v, w \in D \subset \mathbb{C}$.

If $\lambda=\tau \in[0,1]$, then by (74) we get

$$
\begin{aligned}
& \mid \exp z-(1-\tau) \exp v-\tau \exp w \\
& -\sum_{k=1}^{n} \frac{1}{k !}\left[(1-\tau)(z-v)^{k} \exp v+(-1)^{k} \tau(w-z)^{k} \exp w\right] \mid \\
& \leq \frac{1}{(n+1) !} E_{D}\left((1-\tau)|z-v|^{n+1}+\tau|w-z|^{n+1}\right) \\
& \quad \leq \frac{1}{(n+1) !} E_{D} \max \left\{|z-v|^{n+1},|w-z|^{n+1}\right\}
\end{aligned}
$$


for all $z, v, w \in D \subset \mathbb{C}$ and for $\tau=\frac{1}{2}$ we get

$$
\begin{aligned}
& \mid \exp z-\frac{\exp v+\exp w}{2} \\
& \quad-\frac{1}{2} \sum_{k=1}^{n} \frac{1}{k !}\left[(z-v)^{k} \exp v+(-1)^{k}(w-z)^{k} \exp w\right] \mid \\
& \quad \leq \frac{1}{2(n+1) !} E_{D}\left(|z-v|^{n+1}+|w-z|^{n+1}\right)
\end{aligned}
$$

for all $z, v, w \in D \subset \mathbb{C}$.

Moreover, if we take $z=\frac{v+w}{2}$ in (77), then we get

$$
\begin{aligned}
& \mid \exp \left(\frac{v+w}{2}\right)-\frac{\exp v+\exp w}{2} \\
& \\
&-\frac{1}{2} \sum_{k=1}^{n} \frac{1}{2^{k} k !}\left[\exp v+(-1)^{k} \exp w\right](w-v)^{k} \mid \\
& \quad \leq \frac{1}{2^{n+1}(n+1) !} E_{D}|w-v|^{n+1}
\end{aligned}
$$

for all $v, w \in D \subset \mathbb{C}$.

The case $n=0$ gives that

$$
\left|\exp \left(\frac{v+w}{2}\right)-\frac{\exp v+\exp w}{2}\right| \leq \frac{1}{2} E_{D}|w-v|
$$

for all $v, w \in D \subset \mathbb{C}$.

The function $f(z)=\exp z$ is Lipschitzian on $D$ with the constant $L=E_{D}$, then by (52) we get

$$
\left|\exp \left(\frac{v+w}{2}\right)-\frac{\exp v+\exp w}{2}\right| \leq \frac{1}{8} E_{D}|w-v|^{2} .
$$

for all $v, w \in D \subset \mathbb{C}$.

By the convexity in modulus of the complex function and by (66) we also have

$$
\begin{aligned}
& \left|\exp \left(\frac{v+w}{2}\right)-\frac{\exp v+\exp w}{2}\right| \\
& \quad \leq \frac{1}{8}|w-v|\left[\exp (\operatorname{Re} v)+2 \exp \left(\operatorname{Re}\left(\frac{v+w}{2}\right)\right)+\exp (\operatorname{Re} w)\right]
\end{aligned}
$$

for all $v, w \in \mathbb{C}$. 


\section{References}

[1] M. Akkouchi, Improvements of some integral inequalities of H. Gauchman involving Taylor's remainder. Divulg. Mat. 11 (2003), no. 2, 115-120.

[2] G. A. Anastassiou, Taylor-Widder representation formulae and Ostrowski, Grüss, integral means and Csiszar type inequalities. Comput. Math. Appl. 54 (2007), no. 1, 9-23.

[3] G. A. Anastassiou, Ostrowski type inequalities over balls and shells via a Taylor-Widder formula. J. Inequal. Pure Appl. Math. 8 (2007), no. 4, Article 106, 13 pp.

[4] S. S. Dragomir, New estimation of the remainder in Taylor's formula using Grüss' type inequalities and applications. Math. Inequal. Appl. 2 (1999), no. 2, 183-193.

[5] S. S. Dragomir and H. B. Thompson, A two points Taylor's formula for the generalised Riemann integral. Demonstratio Math. 43 (2010), no. 4, $827-840$.

[6] H. Gauchman, Some integral inequalities involving Taylor's remainder. I. J. Inequal. Pure Appl. Math. 3 (2002), no. 2, Article 26, 9 pp. (electronic).

[7] H. Gauchman, Some integral inequalities involving Taylor's remainder. II. J. Inequal. Pure Appl. Math. 4 (2003), no. 1, Article 1, 5 pp. (electronic).

[8] D.-Y. Hwang, Improvements of some integral inequalities involving Taylor's remainder. J. Appl. Math. Comput. 16 (2004), no. 1-2, 151-163.

[9] A. I. Kechriniotis and N. D. Assimakis, Generalizations of the trapezoid inequalities based on a new mean value theorem for the remainder in Taylor's formula. J. Inequal. Pure Appl. Math. 7 (2006), no. 3, Article 90, 13 pp. (electronic).

[10] Z. Liu, Note on inequalities involving integral Taylor's remainder. J. Inequal. Pure Appl. Math. 6 (2005), no. 3, Article 72, 6 pp. (electronic).

[11] W. Liu and Q. Zhang, Some new error inequalities for a Taylor-like formula. J. Comput. Anal. Appl. 15 (2013), no. 6, 1158-1164.

[12] N. Ujević, Error inequalities for a Taylor-like formula. Cubo 10 (2008), no. $1,11-18$.

[13] Z. X. Wang and D. R. Guo, Special Functions, World Scientific Publ. Co., Teaneck, NJ (1989). 
Silvestru Sever DRAGOMIR

Mathematics, College of Engineering \& Science

Victoria University, PO Box 14428

Melbourne City, MC 8001, Australia.

School of Computer Science \& Applied Mathematics,

University of the Witwatersrand, Private Bag 3,

Johannesburg 2050, South Africa.

Email: sever.dragomir@vu.edu.au 